\title{
The dietary approaches to stop hypertension (DASH) diet: Potential for managing hypertension in chronic kidney disease.
}

\author{
Louise M Wells*, Colin H Jones \\ Department of Renal Medicine, York Teaching Hospital NHS Foundation Trust, England
}

Accepted on December 27, 2018

\section{Commentary}

Hypertension is highly prevalent in Chronic Kidney Disease (CKD) and is implicated in renal disease progression. Hypertension is also a major risk factor for Cardiovascular Disease (CVD), the main cause of premature death in the CKD population. Strategies which support management of hypertension are therefore fundamental to effective CKD management. Evidence based guidance on the management of hypertension in CKD focuses on medical and pharmacological approaches, but also highlights the importance of diet and lifestyle modification [1].

The Dietary Approaches to Stop Hypertension (DASH) diet $[2,3]$ promotes an eating pattern based on an increased intake of fruit, vegetables, nuts, seeds, whole grains, fish and dairy produce and decreased intake of sodium. The DASH diet reduces blood pressure in free living adults with normal renal function [3]. In CKD the use of a low sodium diet may augment the efficacy of antihypertensive and anti-proteinuric medications [4].

The evidence base for the DASH diet in populations with normal renal function, when used as initial management or as an adjunct to medication, supports consideration of its use to optimise hypertension management in CKD. Indeed, the DASH diet is advocated by The National Kidney Foundation-Kidney Disease Outcomes Quality Initiative (NKF-KDOQI), alongside other lifestyle modifications, as initial treatment for less severe stages of hypertension in the general population [5]. However individuals with renal insufficiency were excluded from the original DASH studies and an increase in the intake of some of the nutrients advocated by the diet could result in dietary potassium, phosphate and protein contents that exceed those recommended for people with CKD. This has raised concerns around the potential safety of the DASH diet in this setting.

It is well recognised that serum potassium and phosphate can increase as renal function decreases. However, the mechanisms for these increased levels are complex and often multifactorial, including factors such as decreased kidney function, hormonal influences on electrolyte excretion, co-morbid conditions, interaction with other serum components and the prescription of concomitant medications.

Dietary interventions are commonly prescribed to support the management of increased serum potassium and phosphate levels in CKD, but the potential impact on serum concentrations achieved by the dietary modification of these nutrients is also both multifactorial and unpredictable.
A number of recent studies have emphasised the variability in phosphate absorption related to the bioavailability of dietary phosphate according to its chemical form and the food source $[6,7]$. Reduced bioavailability of dietary phosphate, with a potentially minimal effect on serum phosphate levels, is particularly relevant to those foods which are encouraged in the DASH diet, including nuts, seeds and whole grains. There is a theoretical, but less well researched, effect of dietary potassium bioavailability and the impact of different foods on serum potassium levels and its relevance in CKD, and this is an area which warrants further investigation [8].

A recently published study [9] sought to establish the feasibility and safety of using the DASH diet in individuals with CKD. The diet used in the study was based on the intermediate sodium DASH diet, [3] adapted to suit British portion sizes and food choices. Importantly, participants had hypertension and were prescribed inhibitors of the angiotensin system. However, patients with diabetes were excluded, because of their increased risk of hyperkalaemia. The study was small, involving 32 patients, and this is an acknowledged limitation. However, this sample size and the length of follow up is comparable with other published dietary studies in CKD [10] including a pilot study by Tyson et al. [11] which also investigated the potential use of the DASH diet in CKD using a feeding study model.

Feeding studies, where all food is provided to participants over the entire trial period, may be regarded as the gold standard in trials of dietary interventions. This approach can support adherence to the prescribed dietary intake. However a disadvantage of feeding studies is that they do not reflect selfselected dietary intakes in free living subjects. Since the present study aimed to examine the safety and feasibility of using the DASH diet in clinical practice, the application of a 'real life' model of dietary intervention offered the best opportunity to assess acceptability of the diet and therefore its' potential for future therapeutic use. It is acknowledged that this can result in varying adherence to the diet, but reflects the reality of outcomes that may be experienced by free living patients, both in terms of safety and efficacy. It should be noted that while significant changes in dietary intake were achieved in this study, the DASH diet targets for mineral intakes were not actually reached.

Despite the small sample size and failure to fully achieve the target DASH mineral intake, the study demonstrated potentially important clinical outcomes. There was a reduction in dietary intake of sodium and fats, associated with a statistically significant reduction in extracellular fluid volume and systolic and diastolic blood pressure. The level of blood pressure 
reduction observed over the 5 week study follow up period $(9.5$ and $5.3 \mathrm{mmHg}$ for systolic and diastolic blood pressure respectively) could translate into a significant reduction in risk of progression of renal failure and in mortality.

This study suggests that the DASH diet is safe for use in nondiabetic subjects with stage 3 CKD even when antihypertensive medication that blocks the angiotensinaldosterone system is prescribed. The only significant side effects appeared to relate to reduction in blood pressure, which could be appropriately managed by reduction in prescribed antihypertensive medication. The results of the study warrant consideration of a larger population based trial which includes patients with diabetes.

\section{References}

1. National Institute for Health and Care Excellence. Chronic kidney disease: early identification and management of Chronic Kidney Disease in adults in primary and secondary care. NICE guidelines (CG182). 2014.

2. Appel LJ, Moore TJ, Obarzanek E, et al. A clinical trial of the effects of dietary patterns on blood pressure. DASH Collaborative Research Group. $N$ Engl J Med. 1997;336(16):1117-24.

3. Sacks FM, Svetkey LP, Vollmer WV, et al. Effects on blood pressure of reduced dietary sodium and the Dietary Approaches to Stop Hypertension (DASH) diet. DASHSodium Collaborative Research Group. N Engl J Med. 2001;344(1):3-10.

4. Slagman MC, Waanders F, Hemmelder $\mathrm{MH}$, et al. Moderate dietary sodium restriction added to angiotensin converting enzyme inhibition compared with dual blockade in lowering proteinuria and blood pressure: randomised controlled trial. BMJ. 2011;343:d4366.

5. Kidney Disease Outcomes Quality Initiative (K/DOQI). K/ DOQI clinical practice guidelines on hypertension and antihypertensive agents in chronic kidney disease. Am J Kidney Dis. 2004;43(suppl 1):S1-290.

6. Cupisti A, Kalantar-Zadeh K. Management of natural and added dietary phosphorus burden in kidney disease. Semin Nephrol. 2013;33(2):180-90.

7. Williams C, Ronco C, Kotanko P. Whole grains in the renal diet-is it time to re-evaluate their role? Blood Purif. 2013;36(3-4):210-4.

8. St-Jules DE, Goldfarb MD, Sevick MA. Nutrient nonequivalence: Does restricting high potassium plant foods help to prevent hyperkalaemia in haemodialysis patients? J Ren Nutr. 2016;26(5):282-7.

9. Hannah J, Wells LM, Jones CH. The Feasibility of Using the Dietary Approaches to Stop Hypertension (DASH) Diet in People with Chronic Kidney Disease and Hypertension. J Clin Nephrol Kidney Dis. 2018;3(1):1-6.

10. Saran R, Padilla RL, Gillespie BW, et al. A Randomized Crossover Trial of Dietary Sodium Restriction in Stage 3-4 CKD. Clin J Am Soc Nephrol. 2017;12(3):399-407.

11. Tyson CC, Pao-Hwa L, Corsino L, et al. Short term effects of the DASH diet in adults with moderate chronic kidney disease: a pilot feeding study. Clin Kidney J. 2016;9(4): 592-8.

\section{*Correspondence to}

Mrs Louise Wells

Department of Renal Medicine, York Teaching Hospital NHS Foundation Trust

Wigginton Road, York, YO31 8HE, England

Tel: 01904725269

Fax: 01904726867

E-mail: louise.wells@york.nhs.uk 